\title{
From times to worlds and back again: a transcendentist theory of persistence.
}

\begin{abstract}
A. Giordani \& D. Costa
Traditionally, three conceptions of diachronic and diacosmic existence have been presented, that can be roughly characterized as the conjunction of a metaphysical and a semantical thesis.

Endurantism: objects persist through time by being wholly present at each instant of their existence, and different descriptions of an object in time refer to the object itself.

The corresponding modal conception holds that there is a relation of trans-world identity between objects.

Perdurantism: objects persist through time by having temporal parts at each instant of their persistence, and different descriptions of an object in time refer to their temporal parts.

The corresponding modal conception holds that different parts of the same object exist in different worlds.

Exdurantism: objects do not actually persist through time, since they are momentary, i.e. existing in one and only one instant, and different descriptions refer to counterparts of such objects.

The corresponding modal conception holds that different counterparts of the same object exist in different worlds.
\end{abstract}


The intuitive and explicative advantages and problems of these conceptions are well-known. Yet, in the recent literature, endurantism has been subject to a more in-depth analysis, and different versions of it emerged. The present paper aims at disambiguating and completing the debate about diachronic and diacosmic existence (by exploiting the analogy between times and worlds) (§2), and proposing and briefly defending transcendentism - one of the views neglected in the contemporary debate - both in the modal (§3) and the temporal case (§4).

\section{Completing the debate}

\section{$1.1 \quad$ The basic concepts}

Persistence in a dimension $D$ can be thought of as presence at more than one point of it. Hence, we are interested in clarifying how an entity can be present in more points of $D$. In order to do that, we postulate that (i) a dimension be it time or a space of worlds - is characterized by a relation of parthood that conforms to the axioms of classical extensional mereology ${ }^{1}$, and (ii) we introduce the notions of exact location at a region. Location is a relation linking an entity to a region of $D$. Intuitively, an object is generically located at a region when that region is not completely free of it, while it is exactly located at a region when this region is its 'shadow' in the dimension. We shall write " $x @ r$ " for " $x$ is exactly located at $r "$. Let the path of an entity be the sum of all the regions at which it is exactly located [6]:

Definition 1. $\operatorname{path}(x)=\sum_{x @ r} r$, provided $x \in X$

The notion of path allow us to distinguish between proper and derivative

\footnotetext{
${ }^{1}$ It is not difficult to see that a space of worlds can be endowed with a parthood relation by stating that a plurality $P_{1}$ of worlds is part of a plurality $P_{2}$ of worlds when every world in $P_{1}$ is in $P_{2}$. The mereology thus obtained is an atomistic classical extensional mereology, provided we assume that every plurality gives rise to one and only one fusion and that every possible world is an atom in the dimension.
} 
presence. We say that $x$ is properly present in $r$ iff $r<_{D} \operatorname{path}(x)$ and that it is derivatively present in $r$ iff it is it is suitably related to an entity which is properly present at $r$. In drawing this distinction, we allow for the possibility for an entity to be present in a region even if it is not located in it and even if it is not located in any region in $D$.

The notions of exact location and path typically tend to be equated: if an entity has only one exact location, as it is mostly assumed, its only exact location coincides with its path. However, if an entity can have more than one exact location, the two notions are distinct. We will refer to views that hold that an entity has only one exact location as uni-locationist and to views that deny that as multi-locationist [6].

Distinction 1: $\begin{cases}\text { uni-location : } & \forall x, r_{1}, r_{2}\left(x @ r_{1} \wedge x @ r_{2} \rightarrow r_{1}=r_{2}\right) \\ \text { multi-location }: & \exists x, r_{1}, r_{2}\left(x @ r_{1} \wedge x @ r_{2} \wedge r_{1} \neq r_{2}\right)\end{cases}$

In turn, uni-locationism can be differentiated according to how the presence in more than one point is conceived, since a uni-located entity $x$ can be present at two points $r_{1}$ and $r_{2}$ by having parts exactly located there, or not [12, 13, $15]$.

Distinction 2: $\begin{cases}\text { point-location: } & r \ll_{D} \text { path }(x) \rightarrow \exists x_{1}\left(x_{1} \ll_{X} x \wedge x_{1} @ r\right) \\ \text { bare-location: } & r \ll_{D} \text { path }(x) \rightarrow \neg \exists x_{1}\left(x_{1} \ll_{X} x \wedge x_{1} @ r\right)\end{cases}$ In the first case, it can be assumed that the mereological structure of $x$ corresponds to that of the region in which it is located, so that a part of $x$ is exactly located at every part of the exact location of $x$. By contrast, in the latter case, the correspondence between the mereological structure of $x$ and path $(x)$ is broken: $x$ can be exactly located at an extended region $r$, i.e. at a region with proper parts, without having parts that are exactly located at the parts of $r$, 
so that $x$ is both extended, being located at an extended region, and an atom, since it lacks proper parts. Therefore, a theory that admits objects that are both uni-located and properly located in more than one region is assuming the existence of extended atoms (extended simples). We will refer to views that hold that an entity has a part exactly located at any part of its exact location as classical uni-locationist, and to views that allow for extended simples as bare uni-locationist.

Entities can also be derivatively located at more than one point and it is crucial to note that this can occur both (1) when the entity that is derivatively present at a region is properly present at another region of $D$, and (2) when the entity is not properly present at any region $D$, so that it transcends the dimension. A paradigmatic case of entities that are derivatively present in the first way is given by exduring objects, which are derivatively present at regions where they have counterparts. A paradigmatic case of entities that are derivatively present in the second way is given by Plato's ideas, which are derivatively present wherever they are exemplified. Let us call these two ways of being derivatively present counterpart presence and transcendent presence, respectively.

In conclusion, we get the following schema:

$$
\text { presence }\left\{\begin{array}{l}
\text { proper presence }\left\{\begin{array}{l}
\text { classical unilocation } \\
\text { bare unilocation } \\
\text { multilocation }
\end{array}\right. \\
\text { derivative presence }\left\{\begin{array}{l}
\text { counterpart presence } \\
\text { transcendent presence }
\end{array}\right.
\end{array}\right.
$$




\subsection{The debate completed}

The following table clarifies the intuitive relationship between the different ways of being present at more than one region and the theories of diachroinc and diacosmic existence currently discussed.

\begin{tabular}{|c|c|c|}
\hline PROPER PRESENCE & temporal case & modal case \\
\hline \hline classical uni-location & perdurantism & parts theory \\
\hline bare uni-location & extended atoms theory & overlap theory \\
\hline multi-location & multi-locationism & - \\
\hline
\end{tabular}

\begin{tabular}{|c|c|c|}
\hline DERIVATIVE PRESENCE & temporal case & modal case \\
\hline \hline counterpart presence & exdurantism & counterparts theory \\
\hline transcendent presence & - & - \\
\hline
\end{tabular}

Interestingly enough, the table has several empty cells. Let us begin with analyzing and completing the temporal case. As we can see, perdurantism and exdurantism are both unambiguously represented, while endurantism is ambiguously represented in two versions: extended atoms theory and multi-locationism. In the introduction, endurantism was defined as the view according to which objects endure by being wholly present at each instant of their persistence. It is now evident that to be wholly present for $x$ implies having no parts that are exactly located at proper parts of $\operatorname{path}(x)$ and that whole presence can be construed so that either (1) the wholly present object has no part that is exactly located at proper parts of its path, i.e. it is an extended atom, or (2) it is exactly located at every points of its path, i.e. it is multi-located. Accordingly, we obtain the following disambiguations $[6,13,15]$.

Temporal Bare Uni-locationism: objects persist through time by being barely 
located at each instant of their persistence, and different descriptions of an object in time refer to the object itself.

Temporal Multi-locationism: objects persist through time by being exactly located at each instant of their persistence, and different descriptions of an object in time refer to the object itself.

The conception of persistence as transcendent presence at more than one instant is missing. Let us call this view temporal transcendentism. Temporal transcendentism is characterized by the assumption that not every entity is properly present in the temporal dimension. In particular, according to the version we assume, concrete entities can be subdivided into objects and events: events are properly located, more precisely classically uni-located, at times, whereas objects are derivatively present at times, by being partecipants of events. ${ }^{2}$ As a consequence, we define transcendentism about temporal persistence as follows.

Temporal Transcendentism: objects persist through time by taking part in events that are properly located at each instant, and different descriptions of temporal properties of an object refer to different event the object takes part in.

It is important to note that this definition involves a crucial distinction between descriptions of objects simpliciter and descriptions of objects in time. A description of an object in time typically concerns its temporal properties, e.g. being sitting, while a description of an object simpliciter concerns its tenseless properties, e.g. being a man. ${ }^{3}$

\footnotetext{
${ }^{2}$ This interpretation is in accordance with our intuitions concerning objects and events. Indeed, events are taken both in philosophy and in physics to be the principal occupiers of time and most attributions of temporal properties to objects do correspond to events in which such objects take part. To be sure, according to one of the most influential theory of events, temporal exemplifications of properties by objects are to be identified with events.

${ }^{3}$ We are assuming that there is a conceivable distinction between different kinds of properties, mirroring the distinction between accidental and essential properties, but we are not upholding that tenseless properties actually exist. Thus, the previous definition is indepen-
} 
In the modal case several cells have to be filled up. Again, modal perdurantism, i.e. existence through parts, and modal exdurantism, i.e. existence through counterparts [10], are both unambiguously represented. Furthermore, it is possible to define a bare uni-locationist view and a multi-locationist view as follows.

Modal Bare Uni-locationism: objects exist in different worlds by being barely located at each world, and different descriptions of an object in a world refer to the object itself ${ }^{4}$.

Modal Multi-locationism: objects exist in different worlds by being exactly located at each world, and different descriptions of an object in a world refer to the object itself ${ }^{5}$.

Finally, it is possible to define a modal version of transcendentism. Modal transcendentism is characterized by the assumption that not every entity is properly present in the modal dimension and we assume that $U$ and $X$ are defined as before ${ }^{6}$. Thus, while events are classically uni-located at worlds, objects are derivatively present at worlds, i.e. by being partecipants of events.

Modal Transcendentism: objects exist in different worlds by taking part in events that are properly located at each world, and different descriptions of worldly properties of an object refer to different events the object takes part in.

It is worth noting that the previous theses constitute three different construals of the notion of trans-world identity. Moreover, temporal transcendentism is a

dent of the existence of such properties. If no property is tenseless, every description of an object describes the object in time. A defense of tenseless properties is proposed in [5].

${ }^{4}$ This conception seems to have no representant in the current debate.

${ }^{5}$ If we identify modal location with existence in a possible world, then Plantnga's classical modal theory [17] is a kind of modal multi-locationism. See also [14] for a version which shares some intuitions with trascendentism.

${ }^{6}$ However, the events located in other possible worlds will be abstract if you are an abstractionist about possible worlds, while all of them will be concrete if you are a concretist about possible worlds. 
form of endurantism, insofar as it affirms that objects exist at more than one time, thus excluding counterparts presence, without having temporal parts, thus excluding location via parts.

\section{In favor of modal transcendentism}

In this section we will show that a position implying a trans-world identity thesis, $T W I$ for short, is to be preferred relative to the alternative ones. In particular, we will assume that we have strong intuitions concerning how expressions refer to entities and that these intuitions are accounted for by $T W I$, while they are not consistent with its competitors. In addition, we will apply the previous analysis and assess the three ways to construe $T W I$, arguing that the transcendentist interpretation is the more compelling one. Before proceeding, let us say something more on the intuition behind transcendentism. The idea is that concrete objects are constituents of events ${ }^{7}$ and that to exist for an object is to take part in several events, namely those that overlap its history. Therefore, no object exists without having a history and no object can be referred to without reference to a point in that history. As a consequence: (i) objects are not events; (ii) they are not properly in time, even if they are derivatively in time as constituents of events; (iii) they are not properly in the actual world, even if they are derivatively in it, as constituents of their actual history; and (iv) they are not properly in possible worlds, even if they are derivatively in it, as constituents of their possible histories ${ }^{8}$.

\footnotetext{
${ }^{7}$ If you allow for dynamic states of affairs, to say that coincides with saying that objects are constituents of states of affairs. In general, we assume a broad notion of event, including changes and both stationary and momentary events.

${ }^{8}$ What we are providing is just a sketch of a theory of transcendent objects that we consider sufficient to justify their introduction in this philosophical debate.
} 


\subsection{Why prefer $T W I$ : negative grounds}

$T W I$ is to be preferred both for semantical and for metaphysical reasons.

Semantically, the simplest and strongest intuitive argument for $T W I$ is the following. According to our intuitions, it is possible for two propositions both to refer to the same individual and to ascribe incompatible attributes to it, so that it is possible for them to be contradictory, e.g. the following pair of propositions concerning Plato:

1. Plato was sitting when he first met Aristotle.

2. Plato was standing when he first met Aristotle.

If we assume that any proposition is associated with a set of possible worlds, to wit the set of possible worlds where the proposition holds, then it seems straightforward to state that, if a proposition about the individual $x$ holds at a world $w$, then $x$ exists in $w$. We are now able to conclude that $x$ can exist in more than one possible world. Indeed, if $p_{1}$ and $p_{2}$ are two possibly true propositions whose conjunction is impossible, like 1 and 2 , then there are two different possible worlds $w_{1}$ and $w_{2}$ such that $p_{1}$ holds at $w_{1}$ and $p_{2}$ holds at $w_{2}$. Since $p_{1}$ and $p_{2}$ both refer to $x, x$ exists in $w_{1}$ and in $w_{2}$, so that one and the same individual can exist in more than one world. Thus, under the only assumption that the truth at a world of a proposition implies the existence in that world of the individuals the proposition refers to, we can conclude that it is possible for one and the same individual to exist in more than one world. Hence, TWI follows from the assumption that propositions like 1 and 2 are possibly true and refer to the same individual, so that, under the same assumption, we are entitled to deny both that "Plato" refers to different individuals in different possible worlds and that "Plato" refer to different parts of a trans-world individual in different possible worlds. 
Metaphysically, the simplest and strongest intuitive argument for TWI is the following. According to our intuitions, it is possible for the same individual to have different incompatible attributes, so that it is possible for $x$ to be $A_{1}$ and it is possible for $x$ to be $A_{2}$, where $A_{1}$ is incompatible with $A_{2}$. After all, it is not difficult to imagine that Plato, the very same individual that was sitting when he met Aristotle, could have been standing in that very circumstance. On the identity-via-parts theory, there is a trans-world individual that has a part that is $A_{1}$ in the actual world and a different part that is $A_{2}$ in a different world. On the identity-via-counterparts theory, there is an individual that is $A_{1}$ in the actual world and a counterpart of it that is $A_{2}$ in a different world. In both cases, the actual bearer of $A_{1}$, be it the part or the counterpart, is such that it could not have been different. Indeed, since that bearer exists in one and only one possible world, in every possible world in which it exists it is $A_{1}$. Hence, under the assumption that the actual bearer of a property could have been different, we are entitled to deny both that individuals have parts in different possible worlds and that individuals have counterparts in different possible worlds.

These arguments provide support to TWI in the following sense: $T W I$ is surely more intuitive than its competitors; thus, if $T W I$ is both consistent and metaphysically credible, it is to be preferred over them.

\subsection{Why prefer $T W I$ : positive grounds}

Is $T W I$ consistent and metaphysically credible? In section 2 we have noted that there are at least three ways $T W I$ can be interpreted. What we intend to do now is to show that:

- $T W I$ is consistent on any interpretation.

- $T W I$ is metaphysically more credible on the transcendentist interpreta- 
tion.

Consistency. Let $x$ be an individual that is present in more than one world and in such a way that it has neither parts nor counterparts in different worlds. The problem with this conception is that it is ambiguous. Indeed, $x$ can be present in the modal dimension by being present either (1) as something that is derivatively present in that dimension or (2) as something that is properly present in that dimension and, if properly present, it can be present like (2.1) a multi-located atom or like (2.2) a uni-located extended atom. In all cases, the same object can possess different incompatible properties provided it possesses them in different worlds. Thus, there is no problem in assuming that $x$, as existing in $w_{1}$, is $A_{1}$ and that $x$, as existing in $w_{2}$, is $A_{2}$.

Credibility. Let us start with option (2). To say (2.1) that $x$ is a modal multi-located atom is to say that $x$ can be present in more than one possible world and that $x$ can be exactly located in any world in which it is present. To say (2.2) that $x$ is a modal extended uni-located atom is to say that $x$ can be present in more than one possible world and that $x$ can be exactly located in its path through the possible worlds. These options have their own problems. In particular, they seem to be at odds both with a concretist and with an abstractionist conception of possible worlds. Suppose you assume a concretist conception. Then, you should say both that possible worlds are maximal concrete individuals and that you, as a concrete individual, are present in more than one world. Yet, it is hard to see how that can be possible, without saying that you are separated from yourself, i.e. that you are more similar to a flock rather than to an individual. Suppose now you assume an abstractionist conception. Then your path through the possible worlds becomes suspect, because only one world is actual. Thus, if you are a concrete individual in this very world, you 
are a concrete individual in every world in which you exist. Still, according to the abstractionist conception, possible worlds other than the actual one are abstract entities and every possible world exists, even if it is not actual, in every possible world. Therefore, you turn out to be both concrete and abstract, and to have multiple existence in every world, since you are located in every world in every world. As an abstractionist, however, you are allowed to introduce a different entity as a multi-located atom or as an extended uni-located atom, i.e. your essence. If this is your choice, then your position turns out to be safe, since there is no difficulty in assuming that your essence, as an abstract entity, is multi-located or extendedly uni-located. The problem with this move is that you have to interpret modal sentences, that are apparently about you, as sentences stating that abstract entities, such as your essence, are characterized in a certain way. Hence, we hold that an account avoiding this conclusion is to be preferred.

Let us now move to option (1). The idea is that, while transcending the worlds you are present in, you are present in those worlds by supporting events ${ }^{9}$. In our opinion this is the best way to construe the defense of $T W I$ proposed by van Inwagen [18]. This proposal is convincing in showing the consistency of TWI relative to Lewis' criticisms. Nonetheless, some points are left undiscussed and some problems are not addressed. In particular, while objects are said to exist in different possible worlds, nothing is said about the way they exist. In addition, while it is said that the same objects have incompatible properties in different possible worlds it is also said that this peculiar condition is not representable. In fact, all we can represent are objects with different tags on which incompatible properties are recorded, since it is impossible to represent objects having incompatible properties. Still, it seems to be odd to represent a standing Plato and then to tag him as "standing in world 1" and "sitting in

\footnotetext{
${ }^{9}$ In this case, the distinction between abstract and concrete worlds seems to be ineffective.
} 
world 2", while there is nothing odd in representing a standing Plato tagged as "standing" and a sitting Plato tagged as "sitting" as would do both an upholder of the identity-via-parts theory and an upholder of the identity-via-counterparts theory. There is a sense in which the representations produced by the competing theories are truthful, and such sense should be preserved. Transcendentism is able to face both problems. As to the way of existing, a transcendentist can say that objects exist in worlds because they take part in events characterizing them, even if they are not parts of the worlds, thus taking into account the intuition that objects and worlds are different kinds of entities. As to the way of representation, a transcendentist can say that for objects to exist is for them to take part in events, so that to represent objects is to represent them as participants of events, i.e. as inhabitants of possible worlds. Thus, there is no problem in assuming that two representations of Plato are representations of the same object, even if only one of them can represent Plato in the way he actually is.

\section{In favor of temporal transcendentism}

In this final section we briefly argue that transcendentism is to be preferred also as a theory of persistence through time. In particular, besides the arguments for the modal case that directly apply to the temporal case, the following considerations are fundamental.

Apart form transcendentism, all theories of persistence, no matter how different, share two common assumptions, namely that (i) the relation between objects and time is a relation of location, and that (ii) for $x$ to persist is for it to be such that its path is not instantaneous. It is in this sense that multi-location theory, bare uni-location theory and perdurantism affirm, while exdurantism denies, that objects persist though time. By contrast, transcendentism is char- 
acterized by the negation of such assumptions: the relation between objects and time is not a relation of location, but rather an indirect relation definable in terms of their relation with the principal occupiers of time, namely events, and to persist is just to participate in an event whose path is not instantenous. As a consequence, we claim that transcendentism captures the virtues of the other theories, without sharing their problems. In particular, unlike exdurantism, it does not need to deny the phenomenon of persistence and, unlike to perdurantism, it also secures the intuition according to which objects don't need to have temporal parts in order to persist. Furthermore, it exploits the ideas behind multi-locationism, because objects can exist at more instants of time (by participating in instantinous temporal parts of their histories), but it does not share its costs: it does not need to posit that some entities have more than one exact location, postulation that many philosophers believe to be (i) conceptually problematic, given that the only definition of exact location in the literature makes it impossible $[7,16]$, and (ii) paradoxical, given that it clashes with several claims about location that we would usually accept, e.g. the claim that dinstances between regions are iherited by the entities located at them $[1,4$, $6,9]$. Finally, it also exploits the ideas behind extended atoms theory, because objects can exist at intervals of time (by participating in extended temporal parts of their histories), but it does not share its costs, since it does not need to untie the link between extension and possession of parts, which is again, for many philosophers, a conceptual truth $[2,3,7]$.

In conclusion, we believe that the idea that the relation between objects and time passes through their events is an intrinsic virtue of transcendentism, because it accomodates our intuition that events are the principal occupiers of time and the idea that the attribution of temporal features to objects coincides with their events $[8,11]$. In light of all this, transcendentism constitutes a serious 
- though so far neglected - competitor in the debate about the persistence of objects through time.

\section{References}

[1] Stephen Barker and Phil Dowe. Paradoxes of multi-location. Analysis, 63(2):106-114, 2003.

[2] David Braddon-Mitchell and Kristie Miller. The physics of extended simples. Analysis, 66(291):222-226, 2006.

[3] Roberto Casati and Achille C. Varzi. Parts and places: The structures of spatial representation. The MIT Press, 1999.

[4] Douglas Ehring. Spatial relations between universals. Australasian Journal of Philosophy, 80(1):17-23, 2002.

[5] Kit Fine. Tense and reality. modality and tense: philosophical papers, pages 261-320, 2005.

[6] Cody Gilmore. Where in the relativistic world are we? Philosophical perspectives, 20(1):199-236, 2006.

[7] Thomas Hofweber and J. David Velleman. How to endure. The Philosophical Quarterly, 61(242):37-57, 2011.

[8] Jaegwon Kim. Events as property exemplifications. In Action theory, pages 159-177. Springer, 1976.

[9] Shieva Kleinschmidt. Multilocation and mereology. Philosophical Perspectives, 25(1):253-276, 2011.

[10] David K Lewis. On the plurality of worlds. Cambridge Univ Press, 1986.

[11] Lawrence Brian Lombard. Events: A metaphysical study. Routledge and Kegan Paul London, 1986.

[12] Ned Markosian. Simples. Australasian Journal of Philoso- 
phy, 76(2):213-228, 1998.

[13] Kris McDaniel. Extended simples. Philosophical Studies, 133(1):131-141, 2007.

[14] Kris McDaniel. Modal Realism with Overlap. Australasian Journal of Philosophy, 82(1):137-152, 2004.

[15] Josh Parsons. Must a four-dimensionalist believe in temporal parts? The Monist, 83(3):399-418, 2000.

[16] Josh Parsons. Theories of location. Oxford studies in metaphysics, 3, 2007.

[17] Alvin Plantinga. The nature of necessity. Clarendon Press Oxford, 1974.

[18] Peter Van Inwagen. Ontology, identity, and modality: essays in metaphysics. Cambridge University Press, 2001. 